\title{
Injection-Seeded Terahertz Parametric Oscillator with a Ring-Cavity Configuration
}

\author{
Feilong Gao, Yuan Li, Zhenhua Cong *, Xingyu Zhang, Zhaojun Liu, Xiaohan Chen, \\ Zengguang Qin, Zecheng Wang and Na Ming
}

School of Information Science and Engineering, Shandong Provincial Key Laboratory of Laser Technology and Application, Shandong University, Qingdao 266237, China; 201520237@mail.sdu.edu.cn (F.G.);

201511775@mail.sdu.edu.cn (Y.L.); xyz@sdu.edu.cn (X.Z.); zhaojunliu@sdu.edu.cn (Z.L.); cxh@sdu.edu.cn (X.C.); qinzengguang@sdu.edu.cn (Z.Q.); 201920408@mail.sdu.edu.cn (Z.W.); 201920404@mail.sdu.edu.cn (N.M.)

* Correspondence: congzhenhua@sdu.edu.cn

Received: 3 August 2020; Accepted: 20 August 2020; Published: 20 August 2020

check for updates

\begin{abstract}
This paper reports the characteristics of an injection-seeded terahertz parametric oscillator (TPO) with a ring-cavity configuration based on the $\mathrm{MgO}: \mathrm{LiNbO}_{3}$ crystal. The ring cavity is constituted of three mirrors and the $\mathrm{THz}$ wave output surface where the pump and Stokes beams are totally reflected. The $\mathrm{THz}$ pulse energies and the Stokes pulse energies as functions of the pump pulse energy for different seed powers of $47.5 \mathrm{~mW}, 150.7 \mathrm{~mW}$, and $312.8 \mathrm{~mW}$ were investigated. The experimental results showed that the injection-seeded ring cavity TPO exhibited the benefits of lower pump thresholds and higher output energies for the Stokes and THz waves. The smaller the pump pulse energy, the more obvious the effect of the seed injection. The reasons for the laser performance improvement were analyzed.
\end{abstract}

Keywords: terahertz parametric oscillator; nonlinear optics; $\mathrm{LiNbO}_{3}$ crystal; seed injection; ring-cavity

\section{Introduction}

The application and development of terahertz $(\mathrm{THz})$ technology are inseparable from the excellent terahertz radiation sources. Terahertz parametric sources are important terahertz sources and have many advantages such as high peak power, continuous tuning, good temporal and spatial coherence, room temperature operation, compactness, and ease of use [1-3]. Their disadvantages are high pump threshold and low conversion efficiency.

The principle of terahertz parametric sources is the stimulated polariton scattering (SPS) in nonlinear crystals [3,4]. When a pump photon is consumed in the process of SPS, a Stokes photon and a polariton will be generated. The photon energies and the wave vectors obey the energy conservation and the momentum conservation. As the refractive index of the nonlinear crystal in the terahertz range is large, only non-collinear phase matching is available. The terahertz wave tuning can be obtained by adjusting the phase matching angle between the pump and Stokes beams $[5,6]$.

Terahertz parametric sources include terahertz parametric generators (TPGs) and terahertz parametric oscillators (TPOs). A TPG consists of the pump laser source and the nonlinear crystal. If one seed laser is injected into the crystal at the corresponding phase matching angle, the TPG will become an injection-seeded TPG (I-S TPG), whose properties are greatly improved compared with the simple TPG [7-9]. A TPO consists of the pump laser source, the nonlinear crystal, and the Stokes oscillating cavity. The cavity generates a high intensity Stokes beam and determines its propagating direction. The cavity can be a resonant cavity consisting of two mirrors in which the laser beam propagates back and forth along the same axis or a ring cavity having three or more mirrors in which the laser beam propagates in one way [10-16]. A seed can be injected into the Stokes resonant cavity. K. Imai et al. 
reported an extracavity-pumped injection-seeded TPO in 2000. They achieved a narrow $\mathrm{THz}$ wave linewidth $(<200 \mathrm{MHz})$ at a pump pulse energy of $55 \mathrm{~mJ}$ and a seeder power of $90 \mathrm{~mW}$ [17]. In 2009, D. Walsh et al. reported an intracavity-pumped injection-seeded TPO. They achieved continuously tunable $\mathrm{THz}$ wave from $1 \mathrm{THz}$ to $3 \mathrm{THz}$ with the narrow linewidth $(<100 \mathrm{MHz})$ [18]. Both papers focused on the THz wave linewidths of the injection-seeded TPOs. So far, there have been no reports focusing on the improvement of the THz pulse energy based on the ring cavity injection-seeded TPO.

This paper presents an injection-seeded ring cavity TPO based on the $\mathrm{MgO}: \mathrm{LiNbO}_{3}$ crystal. The surface-emitted configuration was used for the THz wave output in this experiment [19]. The ring cavity was constituted of three mirrors and the $\mathrm{THz}$ wave output surface where the pump and Stokes beams are totally reflected. The ring cavity for the injection-seeded TPO has the advantages of easier seed injection and higher pump energies. The experimental results show that the injection-seeded ring cavity TPO exhibits the benefits of lower pump thresholds and higher output energies for the Stokes and $\mathrm{THz}$ waves. The reasons for the laser performance improvement were analyzed.

\section{Experimental Setup}

The experimental setup of the injection-seeded ring-cavity TPO is shown in Figure 1. The $1064.2 \mathrm{~nm}$ pump source was a multi-longitudinal mode Q-switched Nd:YAG laser with a repetition rate of $1 \mathrm{~Hz}$ and a pulse width of $7.5 \mathrm{~ns}$. The pump beam diameter after the aperture was $3.5 \mathrm{~mm}$. Two half wave plates ( 12 ) (Thorlabs Inc., AHWP05M-980, Newton, NJ, USA) and one Brewster plate (BW) (Thorlabs Inc., BW1302, Newton, NJ, USA) were employed to adjust the pump pulse energy and to ensure that the pump laser polarization was parallel to the z-axis of the nonlinear crystal. The ring cavity was constituted by three mirrors M3, M4, and M5, and the THz wave output surface. M4 and M5 were coated for high reflection at the pump and Stokes wavelengths. The transmission of M3 at $1070.2 \mathrm{~nm}$ was $26.4 \%$. The ring cavity length was about $45 \mathrm{~cm}$.

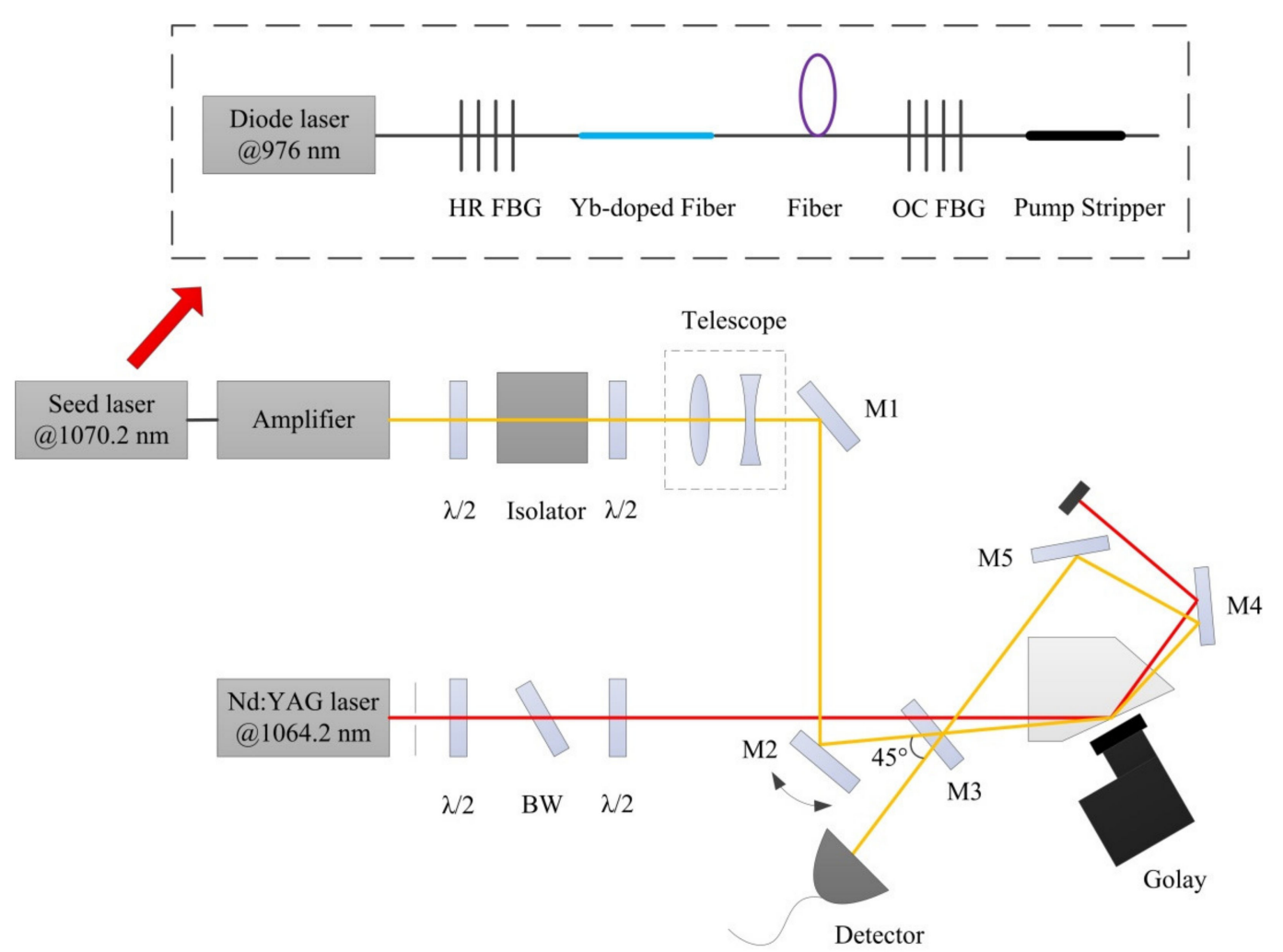

Figure 1. Experimental setup of the injection-seeded ring-cavity terahertz parametric oscillator (TPO).

The seed source was a continuous-wave fiber laser. It was pumped by a diode laser with the wavelength of $976 \mathrm{~nm}$. The output wavelength was $1070.2 \mathrm{~nm}$ and the linewidth was $0.2 \mathrm{~nm}$. The gain 
fiber was $\mathrm{Yb}^{3+}$ doped single mode fiber with a length of $0.5 \mathrm{~m}$. The whole cavity length of the fiber laser was about $10.5 \mathrm{~m}$ after adding a common single mode fiber with a length of $10 \mathrm{~m}$ into the cavity. The cavity length of the Stokes laser was about $45 \mathrm{~cm}$. The long cavity of the seed laser could make the longitudinal modes dense enough, so some of them could be matched with those of the Stokes laser. An isolator (Thorlabs Inc., IO-5-1064-VLP, Newton, NJ, USA) was used to prevent the returned light from damaging the seed laser, and half wave plates $\left(\lambda_{2}\right)$ were used to adjust the polarization of the seed laser to be parallel to the $z$-axis of the nonlinear crystal. We used a telescope to enlarge the seed beam size to make it larger than the pump beam size (enlargement factor was 2.5). The reflectivities of M1 and M2 were higher than $99 \%$ and M2 was mounted on a rotating stage to enable the adjustment of the angle between the Stokes and pump waves. The angle between the injected seed and the output Stokes beam was about $45^{\circ}$, which ensures the safety of the seed laser.

A $5.0 \mathrm{~mol} \% \mathrm{MgO}$ doped $\mathrm{LiNbO}_{3}$ crystal was used as the nonlinear crystal. The angle $\beta$ between the $\mathrm{THz}$ wave and Stokes wave in the $\mathrm{MgO}: \mathrm{LiNbO}_{3}$ crystal was calculated as about $65^{\circ}$ [19]. The shape of the $\mathrm{MgO}: \mathrm{LiNbO}_{3}$ crystal was designed as pentagonal, as shown in Figure 2. The axis $y_{1}$ represents the propagation direction of the terahertz wave. Through this design, the pump and Stokes beams were incident perpendicularly into the crystal and totally reflected on the THz output surface. The generated $\mathrm{THz}$ wave could be emitted vertically through the output surface. The thickness of the $\mathrm{MgO}: \mathrm{LiNbO}_{3}$ crystal was $10.0 \mathrm{~mm}$. The input and output surfaces of the crystal for the pump and Stokes beams were coated with anti-reflection film from $1060 \mathrm{~nm}$ to $1100 \mathrm{~nm}$.

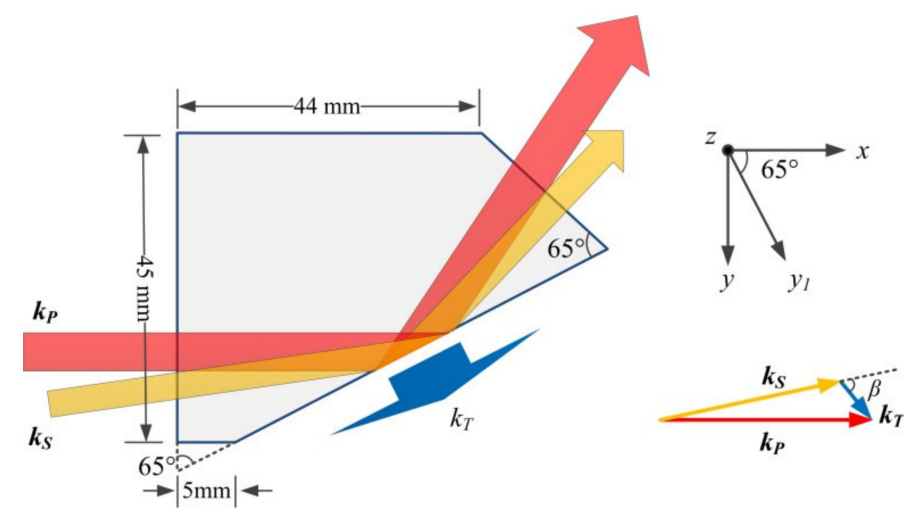

Figure 2. Schematic diagram of the $\mathrm{MgO}: \mathrm{LiNbO}_{3}$ crystal.

The pump and Stokes pulses energies were measured by an energy meter (Coherent Inc., J-50MB-YAG, Santa Clara, CA, USA). The THz signal was detected by a Golay cell (Tydex, GC-1D, Sanit Peterburg, Leningrad Region, Russia). A THz low pass filter (Tydex, LFP14.3-47, Sanit Peterburg, Leningrad Region, Russia) was used to isolate the near infrared wave.

\section{Experimental Results of the Injection-Seeded TPO with the Ring-Cavity}

The angle between the injected seed and the output Stokes wave was designed as approximately $45^{\circ}$. This means that the direction of the Stokes wave output from the cavity was different from that of the seed laser so that the output Stokes wave would not damage the seed laser. By using this ring cavity, the seed injection is convenient. Another advantage of the ring cavity for the injection-seeded TPO is that the nonlinear crystal can endure a stronger pump pulse. For the extra-cavity pumped scheme, the pump pulse propagates through the nonlinear crystal in one-way. Suppose that the pump pulse intensity is $I_{P}$ and the one-way propagating Stokes pulse intensity is $I_{S}$. The total pulse intensity in the ring cavity is $I_{P}+I_{S}$. However, if a conventional Stokes resonator is used, the total pulse intensity in the resonator is $I_{P}+2 I_{S}$ for the same pump intensity.

In order to investigate the effect of the seed power on the TPO performance, three seed powers of $312.8 \mathrm{~mW}, 150.2 \mathrm{~mW}$, and $47.5 \mathrm{~mW}$ (which were measured behind M3) were used in the experiment. The phase matching angle between the pump and Stokes beams outside the nonlinear crystal was 
about $1.5^{\circ}$, at which the generated Stokes wavelength was $1070.2 \mathrm{~nm}$ and the generated $\mathrm{THz}$ wave frequency was $1.58 \mathrm{THz}$. For comparison, the properties of the ring cavity TPO without seed injection were also measured.

Figures 3 and 4 show the THz pulse energies and Stokes pulse energies as functions of the pump pulse energy for different seed powers. The pump threshold was $34.8 \mathrm{~mJ}$ without seed injection. The pump thresholds decreased to $30.3 \mathrm{~mJ}, 26.7 \mathrm{~mJ}$, and $25.2 \mathrm{~mJ}$ with seed powers of $47.5 \mathrm{~mW}$, $150.2 \mathrm{~mW}$, and $312.8 \mathrm{~mW}$, respectively. It can be seen that the pump threshold was going to be saturated with increasing seed powder. Under certain pump energy, both the Stokes and THz pulse energies increased with increasing seed power. When the pump energy was $111.4 \mathrm{~mJ}$, the maximum $\mathrm{THz}$ pulse energy was $1.01 \mu \mathrm{J}$ without seed injection. After injecting the seed for the power of $312.8 \mathrm{~mW}$, the maximum THz pulse energy increased to $1.52 \mu \mathrm{J}$. The corresponding Stokes energies were $12.2 \mathrm{~mJ}$ and $18.6 \mathrm{~mJ}$, respectively.

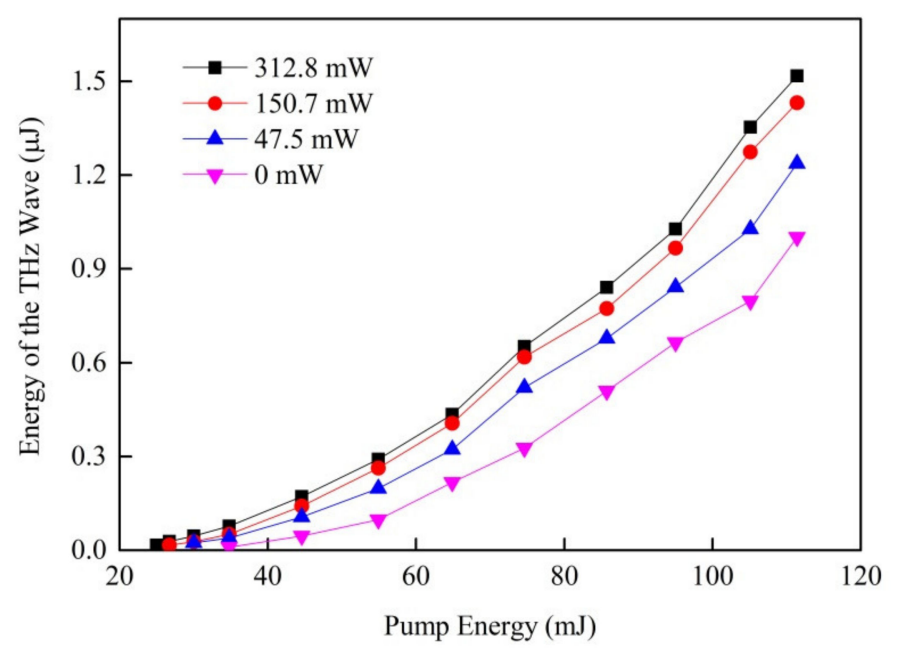

Figure 3. THz pulse energies of the injection-seed ring-cavity TPO with different seed powers as functions of the pump pulse energy.

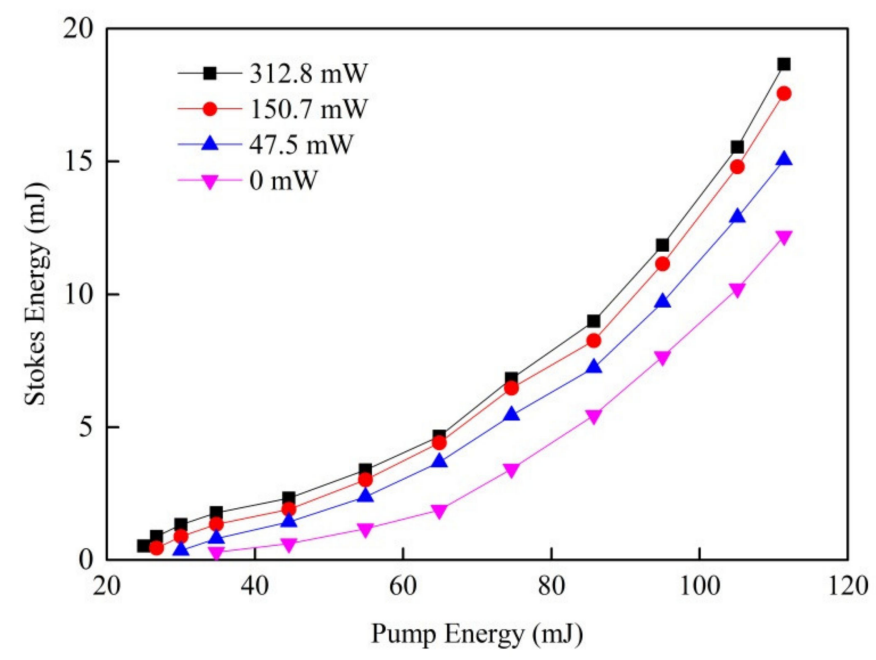

Figure 4. Stokes pulse energies of the injection-seed ring-cavity TPO with different seed powers as functions of the pump pulse energy.

The ratios of $\mathrm{THz}$ energies with seed injection to that without seed injection as functions of the pump pulse energy are shown in Figure 5. When the pump energy was $34.8 \mathrm{~mJ}$, the ratios for the seed powers of $312.8 \mathrm{~mW}, 150.7 \mathrm{~mW}, 47.5 \mathrm{~mW}$ were $6.1,5.1$, and 3.7, respectively. When the pump energy was $111.4 \mathrm{~mJ}$, the ratios were $1.5,1.4$ and 1.2, respectively. The ratio can be considered as an indicator of 
the seed injection effect. It can be concluded that the smaller the pump pulse energy, the more obvious the effect of seed injection. As the seed power increased, the output THz pulse energy was increased, but the growth rate became slower. When the seed power was higher than $312.8 \mathrm{~mW}$, the output energy did not increase significantly, so the maximum seed power was $312.8 \mathrm{~mW}$ in this experiment.

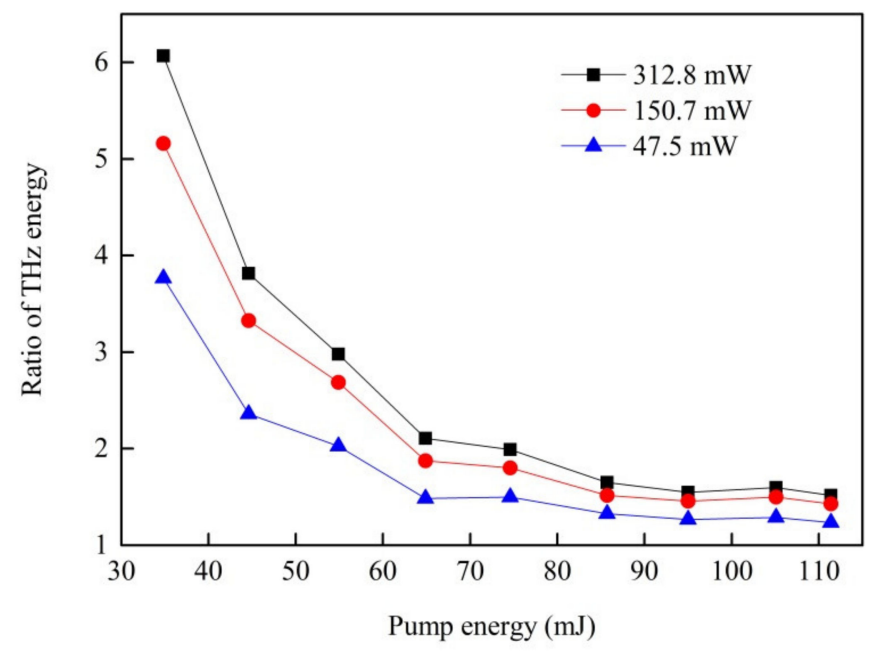

Figure 5. The ratios of $\mathrm{THz}$ energies with seed injection to that without seed injection as functions of the pump pulse energy.

Keeping the pump power of $111.4 \mathrm{~mJ}$, the spectrum of the Stokes wave without seed injection is shown in Figure $6 a$, and Figure $6 b-d$ show the spectra of the Stokes waves for the seed powers of $47.5 \mathrm{~mW}, 150.7 \mathrm{~mW}$, and $312.8 \mathrm{~mW}$, respectively. After the seed injection, the linewidth of the Stokes wave reduced greatly. However, for different seed powers, the spectra of the Stokes waves had little change, and the linewidths kept nearly the same as that of the seed laser (about $0.2 \mathrm{~nm}$ ). This means that after the seed injection, the spectrum of the Stokes wave is mainly determined by the seed laser.
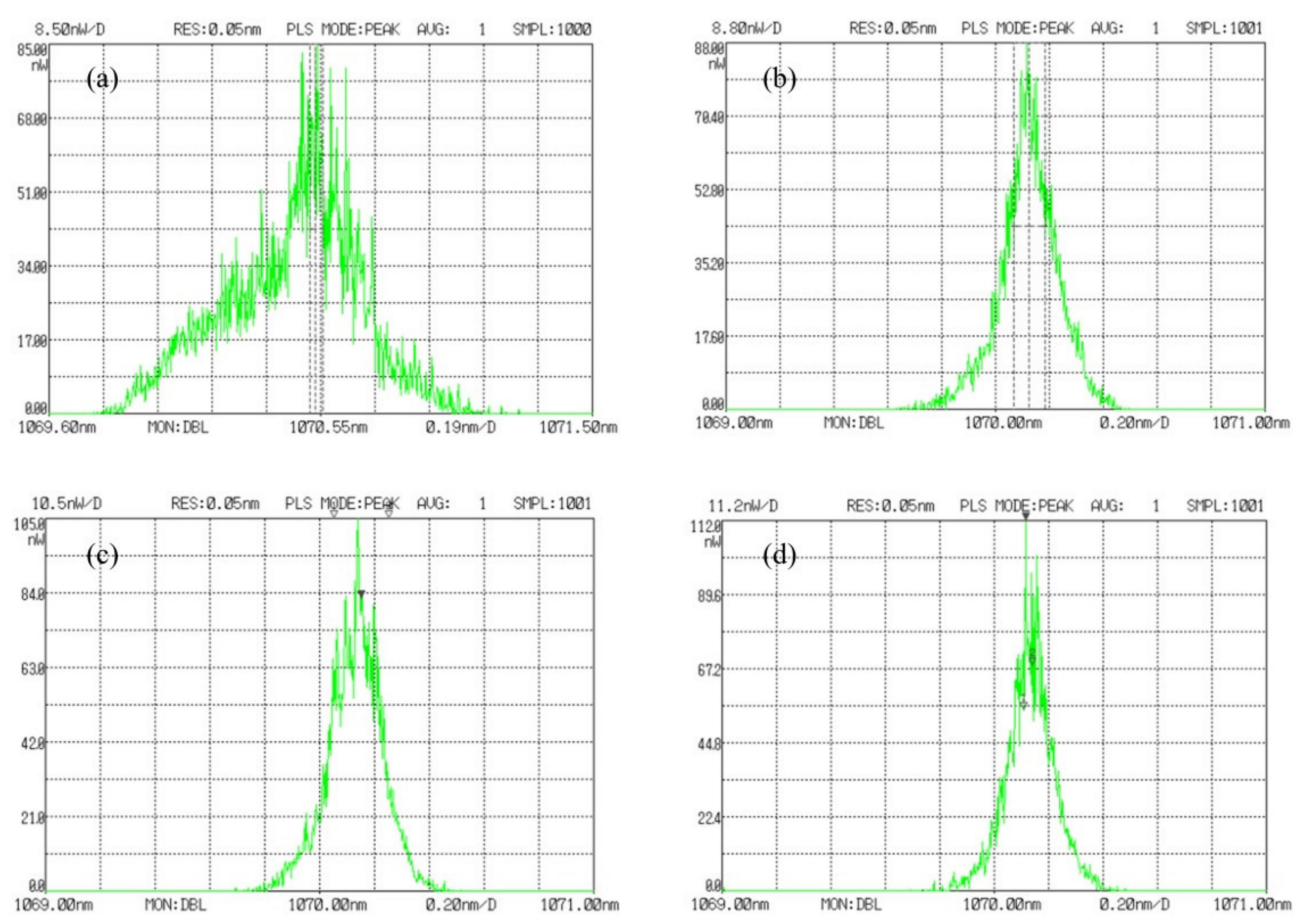

Figure 6. Spectra of the Stokes wave (a) without seed injection, (b) with the seed injection for the power of $47.5 \mathrm{~mW}$, (c) $150.7 \mathrm{~mW}$, and (d) $312.8 \mathrm{~mW}$. 


\section{Discussion}

The process of SPS must obey the energy conservation $\left(\omega_{P}=\omega_{S}+\omega_{T}\right)$ and momentum conservation $\left(\boldsymbol{k}_{P}=\boldsymbol{k}_{S}+\boldsymbol{k}_{T}\right)$, where $\omega_{P}, \omega_{S}$, and $\omega_{T}$ are the frequencies of the pump, Stokes, and THz waves, respectively, and $k_{P}, k_{S}$, and $k_{T}$ are the wave vectors of the three waves. By employing slowly varying envelope approximation, the coupled-wave equations of the $\mathrm{THz}$ wave in the $y$ direction of the nonlinear crystal can be expressed as [20]

$$
\frac{n_{T}}{c} \frac{\partial}{\partial t} I_{T}(x, y, z)+\frac{\partial}{\partial y} I_{T}(x, y, z)=-\alpha_{T} I_{T}(x, y, z)+g \cdot\left(I_{p}(x, y, z) I_{s}(x, y, z) I_{T}(x, y, z)\right)^{1 / 2}
$$

where $I_{i}(i=p, s, T)$ are the intensities of the pump, Stokes, and THz waves, respectively, and $I_{i}$ are the functions of $x, y, z$, and $t$. The absorption coefficient $\alpha_{T}$ and the gain coefficient $g$ are given by

$$
\begin{gathered}
\alpha_{T}=\frac{\omega_{T}^{2}}{k_{T} c^{2}} \operatorname{Im}\left(\varepsilon_{T}\right), \\
g=\frac{n_{T}}{2} \sqrt{\frac{\varepsilon_{0}}{\mu_{0}}} \frac{\omega_{T}^{2}}{k_{T} c^{2}}\left[d_{33}+d_{Q} \operatorname{Re}\left(\varepsilon_{T}-\varepsilon_{\infty}\right)\right]\left(\frac{8}{n_{p} n_{s} n_{T}}\left(\frac{\mu_{0}}{\varepsilon_{0}}\right)^{3 / 2}\right)^{1 / 2},
\end{gathered}
$$

where $\varepsilon_{T}$ is the dielectric constant of the THz wave; $n_{i}(i=p, s, T)$ are the refractive indexes of the nonlinear crystal at the pump, Stokes, and THz wavelengths; $\mu_{0}$ is the permeability of vacuum; $\varepsilon_{0}$ is the permittivity of vacuum; $\varepsilon_{\infty}$ is the high-frequency dielectric constant; $c$ is the light speed in vacuum; and $d_{33}$ and $d_{Q}$ are the nonlinear coefficients that denote the parametric process and Raman process, respectively.

According to Equation (1), the generated THz intensity is related to the product of $I_{P}$ and $I s$. A larger value of the product of $I_{P}$ and $I_{S}$ is profitable for the generation of the THz wave. However, the buildup of the Stokes pulse needs some time, which means that a temporal interval will exist between the pump and Stokes pulses. The seed injection results in a shorter buildup time for the Stokes pulse and a larger product of $I_{P}$ and $I_{S}$, hence profitable for the $\mathrm{THz}$ wave generation. The larger the seed power, the smaller the buildup time will be for the Stokes pulse.

Figure 7 shows the waveforms of the original pump, depleted pump, and Stokes pulses for the pump energy of $111.4 \mathrm{~mJ}$. The waveforms of the pump and Stokes pulses were recorded using two of the same Si detectors (Thorlabs Inc., DET10A/M, 200-1100 nm, rise time $1 \mathrm{ns,} \mathrm{Newton,} \mathrm{NJ,} \mathrm{USA)}$ that could show the temporal relation between the pump and stokes pulses. However, the real pulse intensity was not strictly proportional to the waveform height. The original pump pulse width was 6.9 ns. Figure 7a shows the situation when the seed power was $0 \mathrm{~mW}$ (no seed). The pump pulse was obviously depleted, and the Stokes pulse was built up at which the pump pulse was depleted. The temporal interval between the pump and Stokes pulses was about $7.9 \mathrm{~ns}$. Figure $7 \mathrm{~b}$ shows the situation when the seed power was $47.5 \mathrm{~mW}$. The buildup time of the Stokes pulse was decreased to $6.5 \mathrm{~ns}$. The depletion of the pump pulse was more serious, which means that more pump energy will be transformed into Stokes and THz energy. Figure 7c,d shows the situations when the seed powers were $150.7 \mathrm{~ns}$ and $312.8 \mathrm{~mW}$, respectively. The buildup times of the Stokes pulses were $5.9 \mathrm{~ns}$ and $5.6 \mathrm{~ns}$, respectively. It can be seen that the seed injection leads to the decrease of the temporal interval between the pump and Stokes pulses. The higher the seed power injected, the more pump energy will be consumed to transform into the Stokes wave and $\mathrm{THz}$ wave. 

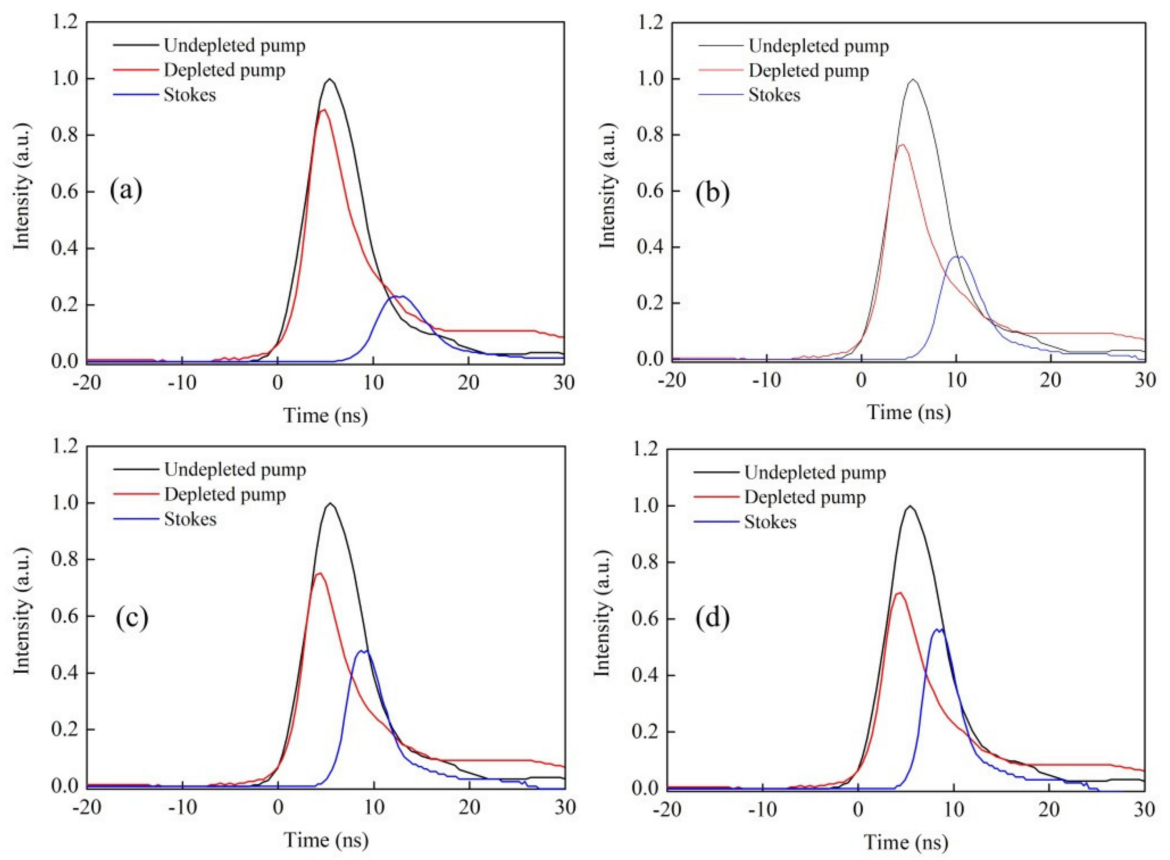

Figure 7. Waveforms of the pump (before and after depletion) and Stokes pulses for the seed powers of (a) $0 \mathrm{~mW}$, (b) $47.5 \mathrm{~mW}$, (c) $150.7 \mathrm{~mW}$, and (d) $312.8 \mathrm{~mW}$.

\section{Conclusions}

The characteristics of the injection-seeded ring-cavity $\mathrm{THz}$ parametric oscillator were investigated. A $5.0 \mathrm{~mol} \% \mathrm{MgO}$ doped $\mathrm{LiNbO}_{3}$ crystal was used as the nonlinear crystal. The pump and seed wavelengths were $1064.2 \mathrm{~nm}$ and $1070.2 \mathrm{~nm}$, respectively. The generated $\mathrm{THz}$ wave frequency was $1.58 \mathrm{THz}$. Compared with the ring cavity TPO without seed injection, the injection-seeded ring cavity TPO exhibited the benefits of lower pump thresholds and higher output energies for the Stokes and $\mathrm{THz}$ waves. The THz pulse energies and the Stokes pulse energies as functions of the pump pulse energy for different seed powers of $312.8 \mathrm{~mW}, 150.7 \mathrm{~mW}, 47.5 \mathrm{~mW}$, and $0 \mathrm{~mW}$ (no seed) were investigated. The pump threshold was $34.8 \mathrm{~mJ}$ without seed injection. The pump thresholds decreased to $30.3 \mathrm{~mJ}$, $26.7 \mathrm{~mJ}$, and $25.2 \mathrm{~mJ}$ with seed powers of $47.5 \mathrm{~mW}, 150.2 \mathrm{~mW}$, and $312.8 \mathrm{~mW}$, respectively. When the pump energy was $111.4 \mathrm{~mJ}$, the maximum $\mathrm{THz}$ wave pulse energy was $1.01 \mu \mathrm{J}$ without seed injection. After injecting the seed for the power of $312.8 \mathrm{~mW}$, the maximum $\mathrm{THz}$ wave pulse energy increased to $1.52 \mu \mathrm{J}$. We found that the smaller the pump pulse energy, the relatively larger effect the injected seed had. The reason for the performance improvement of the injection-seeded ring-cavity TPO was analyzed by using the coupled-wave equations and the temporal interval between the pump and Stokes pulses.

Author Contributions: Conceptualization, F.G. and X.Z.; Methodology, F.G. and Y.L.; Investigation, F.G.; Resources, Z.W. and N.M.; Writing-original draft preparation, F.G.; Writing-review and editing, Z.C., X.Z., and Z.L.; Visualization, X.C. and Z.Q. All authors have read and agreed to the published version of the manuscript.

Funding: This research was funded by the National Natural Science Foundation of China (grant numbers 61475087, 61775122) and the Key Technology Research and Development Program of Shandong (grant numbers 2019JMRH0111, 2017GGX10103).

Conflicts of Interest: The authors declare no conflict of interest.

\section{References}

1. Wu, M.H.; Tsai, W.C.; Chiu, Y.C.; Huang, Y.C. Generation of $\sim 100 \mathrm{~kW}$ narrow-line far-infrared radiation from a KTP off-axis THz parametric oscillator. Optica 2019, 6, 723-730. [CrossRef]

2. Kawase, K.; Sato, M.; Taniuchi, T.; Ito, H. Coherent tunable THz-wave generation from $\mathrm{LiNbO}_{3}$ with monolithic grating coupler. Appl. Phys. Lett. 1996, 68, 2483-2485. [CrossRef] 
3. Kawase, K.; Shikata, J.I.; Ito, H. Terahertz wave parametric source. Phys. D Appl. Phys. 2002, 35, R1-R14. [CrossRef]

4. Johnson, B.C.; Puthoff, H.E.; Hoo, J.S.; Sussman, S.S. Power and linewidth of tunable stimulated farinfrared emission in $\mathrm{LiNbO}_{3}$. Appl. Phys. Lett. 1971, 18, 181-183. [CrossRef]

5. Shikata, J.; Sato, M.; Taniuchi, T.; Ito, H.; Kawase, K. Enhancement of THz-wave output from $\mathrm{LiNbO}_{3}$ optical parametric oscillators by cryogenic cooling. Opt. Lett. 1999, 24, 202-204. [CrossRef] [PubMed]

6. Lee, A.J.; Pask, H.M. Continuous wave, frequency-tunable terahertz laser radiation generated via stimulated polariton scattering. Opt. Lett. 2014, 39, 442-445. [CrossRef] [PubMed]

7. Kawase, K.; Shikata, J.; Imai, K.; Ito, H. Transform limited, narrow-linewidth, terahertz-wave parametric generator. Appl. Phys. Lett. 2001, 78, 2819-2821. [CrossRef]

8. Kawase, K.; Minamide, H.; Imai, K.; Shikata, J.; Ito, H. Injection-seeded terahertz-wave parametric generator with wide tenability. Appl. Phys. Lett. 2002, 80, 195-197. [CrossRef]

9. Wu, M.H.; Chiu, Y.C.; Wang, T.D.; Zhao, G.; Zukauskas, A.; Laurell, F.; Huang, Y.C. Terahertz parametric generation and amplification from potassium titanyl phosphate in comparison with lithium niobate and lithium tantalite. Opt. Express 2016, 24, 25964-25973. [CrossRef] [PubMed]

10. Zhang, R.L.; Qu, Y.C.; Zhao, W.J.; Liu, C.; Chen, Z.L. Si-prism-array coupled terahertz-wave parametric oscillator with pump light totally reflected at the terahertz-wave exit surface. Opt. Lett. 2016, 41, 4016-4019. [CrossRef] [PubMed]

11. Wang, Y.Y.; Ren, Y.C.; Xu, D.G.; Tang, L.H.; He, Y.X.; Song, C.; Chen, L.Y.; Li, C.Z.; Yan, C.; Yao, J.Q. Energy scaling and extended tunability of a ring cavity terahertz parametric oscillator based on $\mathrm{KTiOPO}_{4}$ crystal. Chin. Phys. B 2018, 27, 114213. [CrossRef]

12. Yan, C.; Wang, Y.Y.; Xu, D.G.; Xu, W.T.; Liu, P.X.; Yan, D.X.; Duan, P.; Zhong, K.; Shi, W.; Yao, J.Q. Green laser induced terahertz tuning range expanding in $\mathrm{KTiOPO}_{4}$ terahertz parametric oscillator. Appl. Phys. Lett. 2016, 108, 011107. [CrossRef]

13. Edwards, T.J.; Walsh, D.; Spurr, M.B.; Rae, C.F.; Dunn, M.H.; Browne, P.G. Compact source of continuously and widely-tunable terahertz radiation. Opt. Express 2006, 14, 1582-1589. [CrossRef] [PubMed]

14. Ortega, T.A.; Pask, H.M.; Spence, D.J.; Lee, A.J. THz polariton laser using an intracavity $\mathrm{Mg}_{\mathrm{LiNbO}} \mathrm{Linstal}_{3}$ with protective teflon coating. Opt. Express 2017, 25, 3991-3999. [CrossRef] [PubMed]

15. Minamide, H.; Ikari, T.; Ito, H. Frequency-agile terahertz-wave parametric oscillator in a ring-cavity configuration. Rev. Sci. Instrum. 2009, 80, 123104. [CrossRef] [PubMed]

16. Yang, Z.; Wang, Y.; Xu, D.; Duan, P.; Yan, C.; Tang, L.; Yao, J. High-energy terahertz wave parametric oscillator with a surface-emitted ring-cavity configuration. Opt. Lett. 2016, 41, 2262-2265. [CrossRef] [PubMed]

17. Imai, K.; Kawase, K.; Shikata, J.; Minamide, H.; Ito, H. Injection-seeded terahertz-wave parametric oscillator. Appl. Phys. Lett. 2001, 78, 1026-1028. [CrossRef]

18. Walsh, D.; Stothard, D.; Edwards, T.; Browne, P.; Rae, C.; Dunn, M. Injection-seeded intracavity terahertz optical parametric oscillator. J. Opt. Soc. Am. B 2009, 26, 1196-1202. [CrossRef]

19. Ikari, T.; Zhang, X.B.; Minamide, H.; Ito, H. THz-wave parametric oscillator with a surface-emitted configuration. Opt. Express 2006, 14, 1604-1610. [CrossRef] [PubMed]

20. Wang, W.T.; Zhang, X.Y.; Wang, Q.P.; Cong, Z.H.; Chen, X.H.; Liu, Z.J.; Qin, Z.G.; Li, P.; Tang, G.Q.; Li, N.; et al. Multiple-beam output of a surface-emitted terahertz-wave parametric oscillator by using a slab $\mathrm{MgO}: \mathrm{LiNbO}_{3}$ crystal. Opt. Lett. 2014, 39, 754-757. [CrossRef] [PubMed]

(C) 2020 by the authors. Licensee MDPI, Basel, Switzerland. This article is an open access article distributed under the terms and conditions of the Creative Commons Attribution (CC BY) license (http://creativecommons.org/licenses/by/4.0/). 